\title{
Benign Versus Malignant Cervical Lymph Nodes ; Differentiation by Diffusion Weighted MRI
}

\author{
Mohamed A. Mohammad, Tamer A. Kamal, Haney H. Lotfy
}

Department of diagnostic and interventional radiology, Benha faculty of medicine, Banha University, Egypt.

Correspondence to: Mohamed A. Mohammad, Department of diagnostic and interventional radiology, Benha faculty of medicine, Banha University, Egypt.

Email:

Mohamed.azamsoliman@gm ail.com

Received: 14 December 2019

Accepted: 11 December 2021

Abstract : Cervical adenopathy is a common problem and the differentiation of benign and malignant node is of crucial importance for therapy management. Objective: This prospective study aimed to know if Diffusion weighted images (DWI) and apparent diffusion coefficient (ADC) can differentiate benign from malignant cervical lymphadenopathy. Patients and methods: Thirty patients with cervical adenopathy were included in this study. Doppler ultrasound, DWI and ADC maps were automatically reconstructed and used for the measurement of ADC values. The approval of the institutional ethical committee was obtained before beginning work. Results: The sensitivity and specificity of the RI cut-off value $<0.69$ in differentiation between benign and malignant cervical L.Ns was $88.8 \%$ and $71.4 \%$, respectively. The optimal ADC cut off value for differentiation between benign and malignant lymph nodes was $61.0 \cdot 103 \mathrm{~mm} 2 / \mathrm{s}$ with an accuracy $96.7 \%$, a sensitivity $100 \%$, a specificity $88.9 \%$, PPV 95.4\% and NPV 100\% and statistically significant P-value $=0.000$.

Conclusion: DWI and ADC were useful for differentiation between benign and malignant cervical lymphadenopathy and recommended to decrease the need of invasive biopsies. However, CDUS techniques can be used as preliminary technique but, they had potential pitfalls in diagnosis of malignant cervical lymphadenopathy cases.

Keywords: Diffusion weighted image (DWI); Apparent diffusion coefficient (ADC); Lymphadenopathy.

\section{Abbreviations:}

DWI: Diffusion weighted imaging.

ADC: Apparent diffusion coefficient.

CDUS: Color doppler ultrasonography 


\section{Introduction:}

Cervical lymph nodes are prone to be involved in a number of pathologic processes. They are common sites for lymphoma, metastasis, and reactive enlargement in a number of conditions including tuberculosis (TB) (1)

Differentiation between benign and malignant lymph nodes usually changes the prognosis of the patient and plays a crucial role in planning the therapy of malignancy suspected patients (2). However, the differentiation between benign and malignant lymph nodes remains challenging .(3)

Color Doppler ultrasound has been proposed as a method of differentiating benign from malignant lymphadenopathy, as it is a sensitive non-invasive imaging technique capable of detecting vessels as small as those found in lymph node. (7)

Diffusion-weighted MR imaging (DWI) is a non-invasive functional technique and valuable tool for the identification and characterization of lymph nodes; it highlights both normal and pathologic lymph nodes and enables measuring diffusion in lymph nodes by means of the so-called apparent diffusion coefficient
(ADC). Therefore, DWI can be helpful in distinguishing between causes of cervical lymphadenopathy. (9)

Normal lymph nodes have a relatively restricted diffusion (low ADC) because of their high cellular density. Metastatic lymph nodes may have increased cellular density or necrotic areas, which further restricts or increases diffusion, respectively.(6)

\section{Patients and Methods:}

Thirty consecutive patients presenting with enlarged neck lymph nodes were included in this prospective study, during the period from January 2017 and March 2019. Their age ranged from 11 to 65 years (mean age was 38.5 years), 21 were males while only 9 female cases were found. Patients were referred to MRI unit of the Maadi Armed forces hospitals after clinical detection of enlarged cervical L.N groups. Only 6 cases had signs of inflammation.

\section{Inclusion and exclusion criteria:}

Patient presented with cervical lymphadenopathy regardless of age or sex. Cases with previous surgery or radiotherapy were excluded. Written informed consent was obtained from all patients and the study 
was approved by local ethics committee. All cervical L.N groups were involved in this study, one L.N was examined in 20 cases, 2 L.Ns were examined in the other 10 cases. The final suggested diagnosis was made by three radiologists. They were aware of the clinical data, then the imaging findings were correlated with the pathological data. The patients with neck nodal disease underwent the following:

\section{(1) Clinical assessment:}

Full history and clinical examination: of the enlarged L.Ns, performed by referring physicians.

\section{(2) Grey scale and color Doppler ultrasound examination:}

Gray scale and Doppler sonography using linear transducer (frequency $7.5 \mathrm{MHz}$ ) at ultrasound system of Logic 7.

(A) Grey scale ultrasound examination: the largest lymph nodes were assessed for the Size: Long and short axis diameters were measured. Shape: The shape of the lymph node was determined by the short axis to long axis ratio $(\mathrm{S} / \mathrm{L})$. A $\mathrm{S} / \mathrm{L}$ ratio less than 0.5 indicates a long or oval node, whereas greater than or equal to 0.5 indicates a round node. Nodal hilum: wide, narrow or absent. Echogenicity: hypoechoic, hyperechoic or isoechoic. Homogeneity: homogenous or heterogeneous. Nodal border: either well or ill defined border. Ancillary features: including cystic necrosis, calcification, matting and surrounding soft tissues.

(B) Color Doppler ultrasound examination: If a vascular signal was detected in a node (hilar, peripheral, mixed, intranodal dots), spectral Doppler was performed to measure the vascular resistance. The sample volume was standardized to $1 \mathrm{~mm}$ and was placed in the center of the vessel. Angle correction was made at an angle of 60 or less in all examinations. Each measurement of RI was obtained from three consecutive Doppler spectral waveforms or from the clearest arterial signal.

\section{(3) MRI imaging:}

All patients were examined on 1.5T Philips superconducting MR imager (Achieva, class IIa , USA).

1. Conventional non contrast MR imaging: Preliminary to the diffusion weighted magnetic resonance imaging, including Axial T1WI and T2WI.

2. Diffusion weighted magnetic resonance imaging: Single-Shot Echo-planar diffusion sensitized sequences (DWI) (TR $3.4 \mathrm{~s}$, TE $99 \mathrm{~ms}$, Matrix 512 · 512, slice-thick- ness 4 $\mathrm{mm}$ with an interslice gap of $1 \mathrm{~mm}$ and 
FOV $230 \mathrm{~mm}$ ) were acquired on the axial plane.

The diffusion-sensitizing gradients were applied with a b value of 0 and $1000 \mathrm{~s} / \mathrm{mm}^{2}$ per axis in each patient. The b-value used in this study has been determined by the specifications of the MR-scanner and the image quality. There is no unique b-value established for DWI of lymph nodes yet. ADC maps were automatically reconstructed for all diffusion-weighted images and used for the measurement of ADC values.

Isotropic diffusion images with $\mathrm{b}=$ $1000 \mathrm{~s} / \mathrm{mm} 2$ were generated from the three diffusion directions assessed. Trace ADC maps were generated automatically.

Small (ROI) was drawn in most of exams; solid adenopathies were evaluated placing an ROI over the examined lymph node while in necrotic adenopathy, if obvious solid and necrotic components are visible, ROIs were placed on separate tissue portions.

\section{(4) Histopathological correlation:}

Final diagnosis for all lymph nodes was made by correlation between color doppler sonographic findings, magnetic resonance diffusion readings and histopathological sections which were performed in 30 cases.
FNAB was done in 9 cases which were suspected to be inflammation and excision biopsy in 24, three of them had inadequate samples by FNAB.

\section{Statistical analysis:}

According to the histopathological analysis of the enlarged lymph nodes, our patients were divided into benign (including inflammatory and granulomatous) and malignant (including metastasis and lymphoma). Statistical analysis was done using SPSS version 17 ("'Statistical Package for Social Science"). The mean for RI and ADC was calculated for each group. Oneway (ANOVA) test was done to compare results of more than two groups and student's t-test to compare results between two groups. P value of less than 0.05 was considered to indicate a statistically significant difference. The RI and ADC cutoff value for differentiating malignant from benign nodes was determined using Kappa test. Diagnostic accuracy of both color Doppler ultrasound and diffusion weighted MRI was evaluated in terms of sensitivity, specificity, positive predictive value (PPV), and negative predictive value (NPV). 


\section{Results:}

In this study enlarged cervical L.Ns was found more in male patients (21 cases, 70\%) and 9 cases were females (30\%). Their ages ranged from 11 to 65 years, Mean age was 38.5 years. Pathological examination of the studied lymph nodes in the examined 30 patients revealed: benign cases ( 5 cases of inflammatory, 3 cases granulomatous), 12 lymphomatous cases including (9 cases of Non-Hodgkin Lymphoma and 3 cases of Hodgkin Lymphoma) and 9 metastatic cases.

Regarding the LNs size, the mean $\mathrm{S} / \mathrm{L}$ ratio of malignant LNs was 0.66 and 0.63 for the 1ry and 2ry malignant L.Ns (Figs. 1 and 2 , respectively), which was higher than mean $\mathrm{S} / \mathrm{L}$ ratio of benign $\mathrm{LNs}$ as it was 0.42 and 0.44 for the inflammatory and granulomatous L.Ns (Figs. 3 and 4, respectively). Gray scale US features showing benign lymph nodes in our study had preserved oval shape in all inflammatory cases and in 2/3 granulomatous cases. Additionally preserved wide central hilum, displaying homogenous hypoechoic echogenicity, with well defined borders (Table 1). On the other hand most of rounded L.N shape was found in 7/12 (58.3\%) with lymphomatous cases and 6/9 metastatic lymph nodes cases, moreover the $\mathrm{S} / \mathrm{L}$ ratio was $>0.5$ for all malignant cases, with abnormally narrow or lost hilum, dis- playing heterogeneous hypoechoic echogenicity, with well defined borders. The ancillary features in general were more frequently seen in malignant than in benign lymph nodes except soft tissue edema which was detected in association with the Calcification was found in $3 / 9$ cases $(33.3 \%)$ of metastatic L.Ns and 2/12 (16.6\%) of lymphomatous L.Ns, regarding intranodal necrosis, it was more frequently seen in malignant LNs in $8 / 12$ cases $(66.6 \%)$ of 1 ry malignant LNs and $3 / 9$ cases $(33.3 \%)$ of 2ry malignant LNs, while none of the benign lymph nodes showed calcifications or intra-nodal necrosis. Matting was present in 3/9 case $(33.3 \%)$ and in $2 / 12(22.2 \%)$ of 1ry and 2ry malignant LNs, respectively, while soft tissue edema was seen only in 4/6 cases $(66.6 \%)$.

In the current study the vascular pattern (Table 2) was as follows, most of the lymphomatous lymph nodes showed mixed flow in 7/12 cases $(58.3 \%$ ) (Fig. 1), while metastatic cases showed peripheral flow in $4 / 9$ cases $(44.4 \%)$ and mixed in 2/9 cases (22.2\%) (Fig. 2). Hilar vascularity was found in all inflammatory lymph nodes 
in $6 / 6$ cases $(100 \%)$ (Fig. 3), as well as most of granulomatous lymph nodes in $2 / 3$ cases $(66.6 \%)$ (Fig. 4). Only in one case (33.3\%) with granulomatous (tuberculous) lymphadenopathy; matted LNs showed mixed vascular pattern of perfusion. A vascular pattern is found only in one metastatic L.N case (11.1\%). This difference was statistically significant with P-value = 0.004. Based on vascular pattern alone color Doppler showed an accuracy $83.3 \%, \quad$ sensitivity $=100 \%, \quad$ specificity $=$ $76.2 \%, \mathrm{PPV}=64.3 \%$ and $\mathrm{NPP}=100 \%$.

The mean RI of benign lymph nodes was 0.59 , and significantly lower than mean RI of malignant lymph nodes (0.78), (P-value $=0.001)$.

Statistical analysis of our results showed that RI cut off value for differentiation between benign and malignant lymph nodes is 60.69 with an accuracy $=76.7 \%$, sensitivity $=88.8 \%, \quad$ specificity $=71.4 \%$, $\mathrm{PPV}=57.1 \% \quad$ and NPP $=93.7 \%$ and $\mathrm{P}-$ value $=0.002$.

The benign lymph nodes (9/9) 100\% appeared hyperintense in $(b=0)$ DW images, hypointense in $(b=1000) \mathrm{DW}$ images and hyperintense in ADC maps, also all malignant lymph nodes (21/21) appeared hyperintense in both $(b=0)$ and $(b=1000)$ DW images and hypointense in $\mathrm{ADC}$ maps.

The malignant lymph nodes in this study showed lower mean ADC value (Figs. 1 and 2) than benign lymph nodes (Figs. 3 and 4), this difference was highly statistically significant because $\mathrm{P}$-value = 0.000 (Table 3).

We found that the Non Hodgkin lymphomatous nodes had lower ADC values (mean $0.69 \cdot 103 \mathrm{~mm} 2 / \mathrm{s}$ ) (Fig. 1), than Hodgkin lymphomatous nodes (mean $0.81 \cdot 103 \mathrm{~mm} 2 / \mathrm{s}$ ), however this difference was not statistically significant because $\mathrm{P}$-value $=0.12$.

In our study there were 13/21 malignant lymph node cases (6 lymphomatous nodes and 7 metastatic nodes) that showed necrotic areas of high signal intensity on both T2W images and $(\mathrm{b}=0)$ DW images, of low signal in $(b=1000) \mathrm{DW}$ images and high signal intensity on ADC maps. The ADC values were calculated from the solid and necrotic parts of the lymph nodes separately. The above table shows that $\mathrm{ADC}$ values measured in the nodal necrotic areas were higher than ADC values measured in nodal non- 
Benha medical journal, vol. 39, special issue (radiology), 2022

necrotic or solid areas in both 1ry

differentiation between benign and malignant and 2ry malignant lymph nodes malignant lymph nodes is $61.0 \cdot 10 \quad 3$ with significant P-values (0.000 and 0.01, $\mathrm{mm} 2 / \mathrm{s}$ with accuracy $96.7 \%$, sensitivity respectively).

Statistical analysis of our results showed that optimal ADC cut off value for 100\%, specificity $88.9 \%$, PPV $95.4 \%$ and NPV $100 \%$ and highly significant P-value $=0.000$. inflammatory lymph nodes $(4$ nodes, 66.6\%) (Table 1).

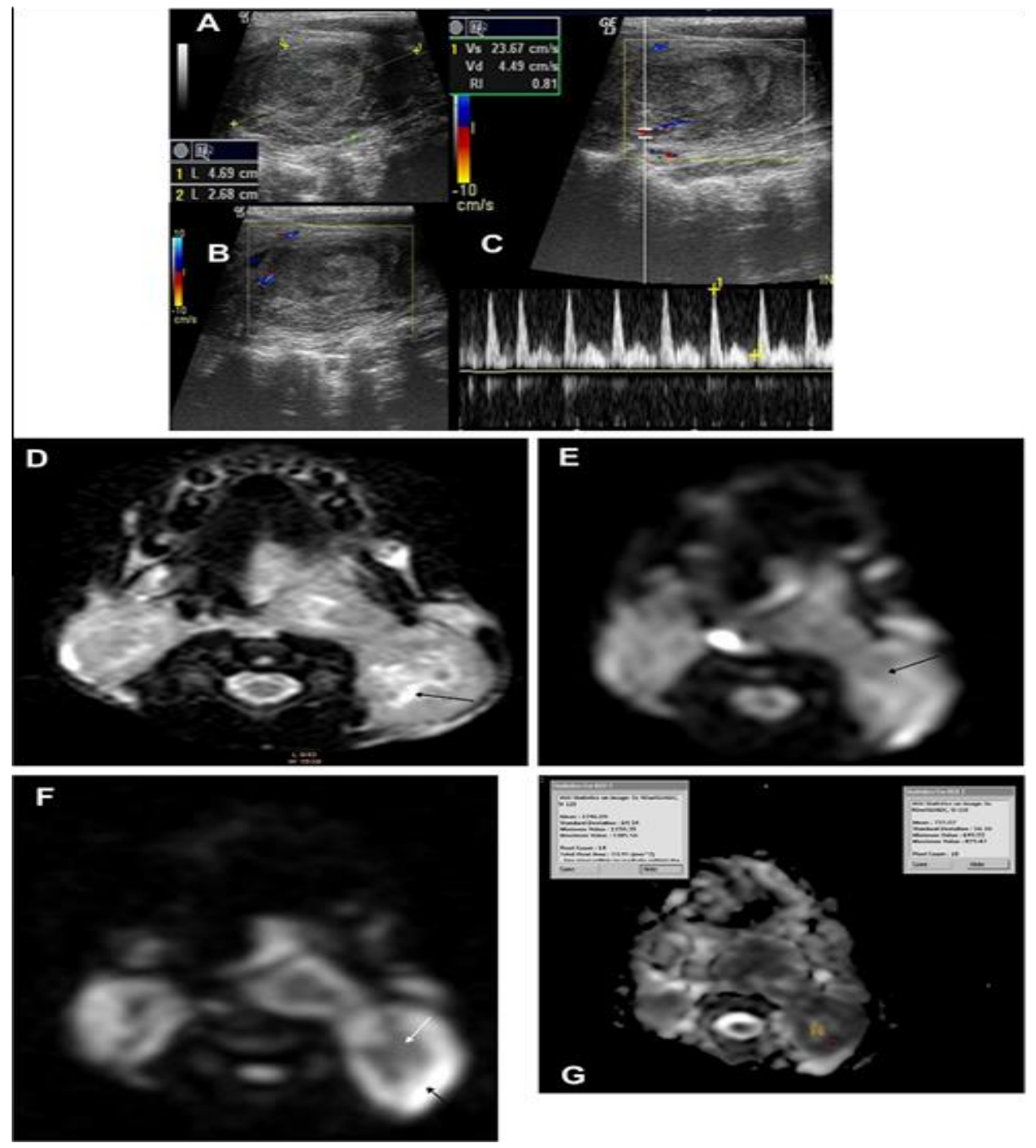

Fig. 1 (A-C): Grey scale, Color Doppler US images of the studied L.N; reveal enlarged rounded $(\mathrm{S} / \mathrm{L}$ ratio $>0.5)$ heterogeneous echo pattern with peripheral vascularity pattern of perfusion, mean RI $=0.81$. (D) Axial T2W-MR image of the studied L.N; reveals enlarged left posterior cervical lymph node at level VA that exhibits high signal intensity (arrow). (E) DW-MR image at $(b=0)$ of the studied L.N: show that the lymph node appears hyperintense with small hypointense areas of necrosis (arrow). (F) DW-MR image at $(\mathrm{b}=1000)$ of the studied L.N: show that the lymph node's solid part appear hyperintense (black arrow) with hypointense areas of necrosis (white arrow). (G) ADC map of the studied L.N: showing low signal intensity of the studied lymph node. The mean ADC value of the solid part $(0.73 \cdot 103 \mathrm{~mm} / \mathrm{s})$ and ADC value of necrotic part (1.24 $103 \mathrm{~mm} 2 / \mathrm{s}$ ). The studied images findings suggestive of malignant disease (Non-Hodgkin lymphoma). 


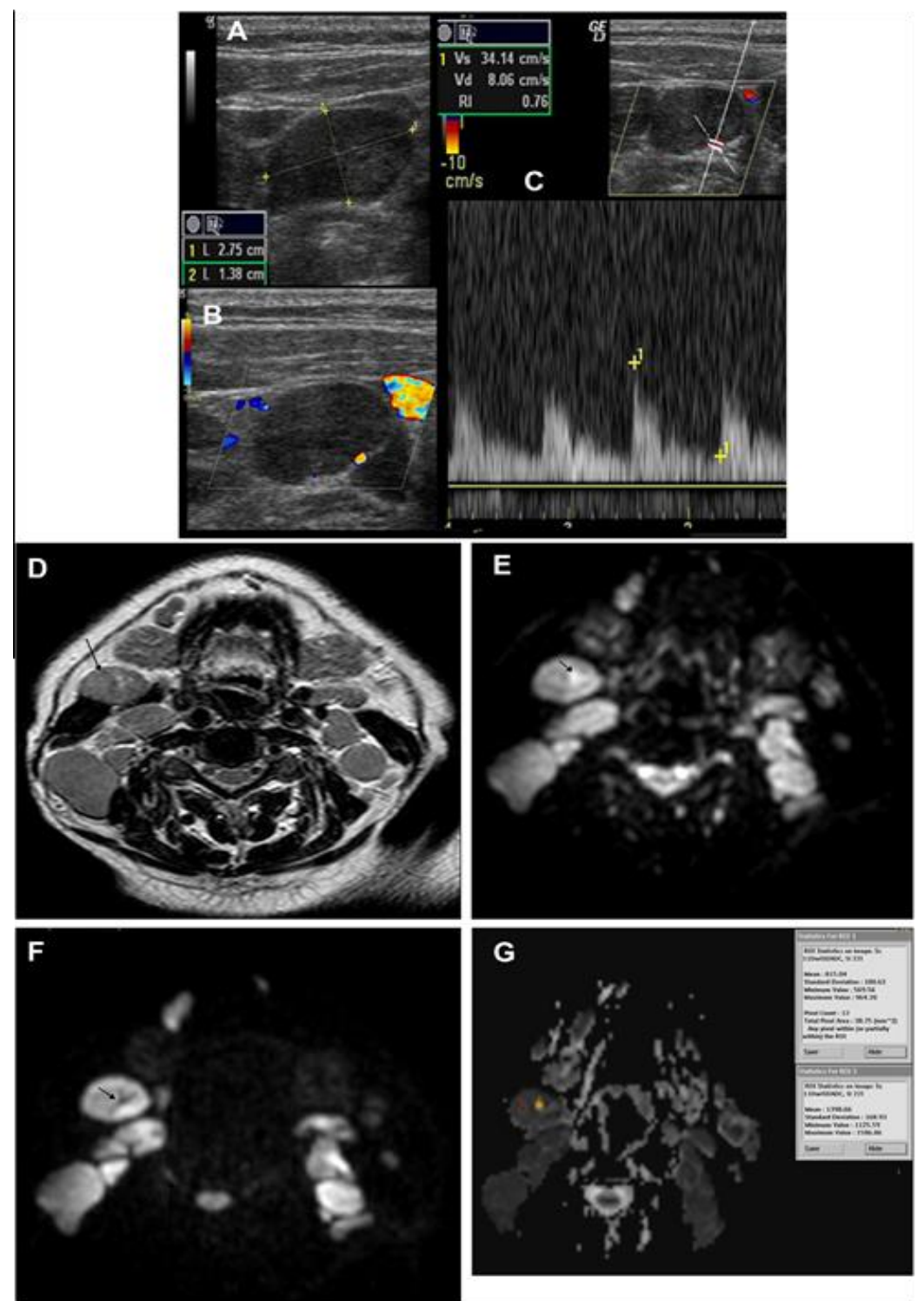

Fig. 2 (A-C): Grey scale, Color and pulsed Doppler US images of the studied L.N; reveal oval shape (S/L ratio > 0.5), hypoechoic $\mathrm{LN}$ with peripheral pattern of perfusion with $\mathrm{RI}=(0.76)$. (D) Axial T1W-MR image of the studied L.N: reveals enlarged right upper internal jugular lymph node that exhibits heterogeneous high signal intensity (arrow). (E) DW-MR image at $(b=0)$ of the necrotic studied L.N: shows that the lymph node appears hyperintense with more hyperintense small areas of necrosis (arrow). (F) DW-MR image at $(b=1000)$ of the studied L.N: shows that the lymph node appears hyperintense with small areas of necrosis displaying hypointense signal (arrow). (G) ADC map of the studied L.N: showing low signal intensity of the lymph. The mean ADC value of the nodal solid region is $(0.81 \cdot 103 \mathrm{~mm} 2 / \mathrm{s})$ and of nodal necrotic region is $(1.39 \cdot 103 \mathrm{~mm} / \mathrm{s})$. The findings suggestive of malignant disease (metastatic malignant follicular carcinoma). 

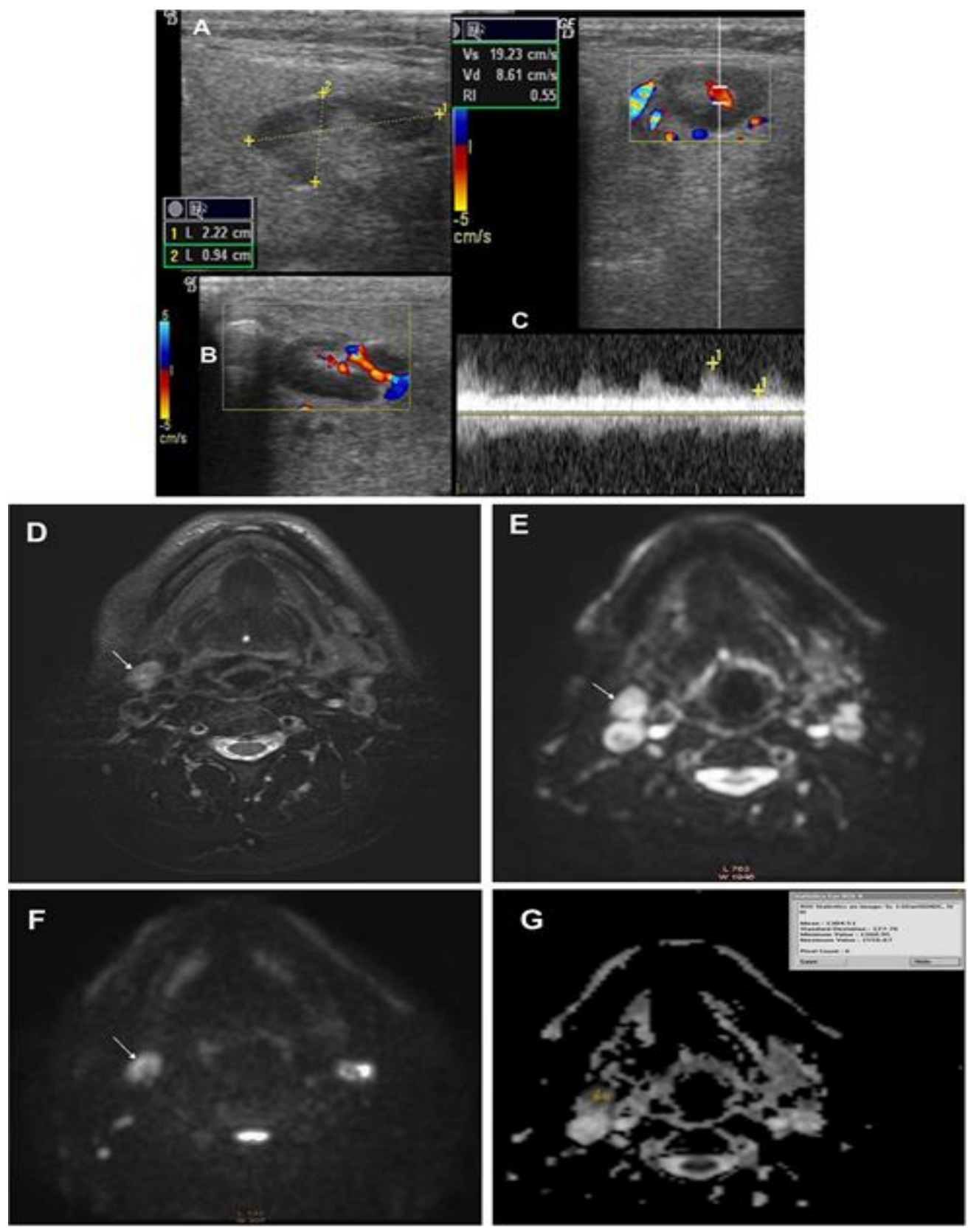

Fig. 3 (A-C): Grey scale, Color and pulsed Doppler US images of the studied L.N; reveal oval shape enlarged LN (S/L ratio $<0.5$ ), and preserved normal central echogenicity with central hilar pattern of perfusion with $\mathrm{RI}=0.55$. (D) Axial STIR MR image of the studied L.N: reveals enlarged right upper internal jugular lymph node at level IIA that exhibit high signal intensity (arrow). (E) DW-MR image at $(\mathrm{b}=0)$ of the studied L.N: shows that the lymph node appears hyperintense (arrow). (F) DW-MR image at $(\mathrm{b}=1000)$ of the studied L.N: show that the lymph node appears hypointense (arrow). (G) ADC map of the studied L.N: showing high signal intensity of the lymph node. The mean ADC value of the studied lymph node is $(1.38 \cdot 10 \quad 3 \mathrm{~mm} 2 / \mathrm{s})$. The imaging findings suggestive of benign disease (Chronic non specific reactive lymphadenitis). 


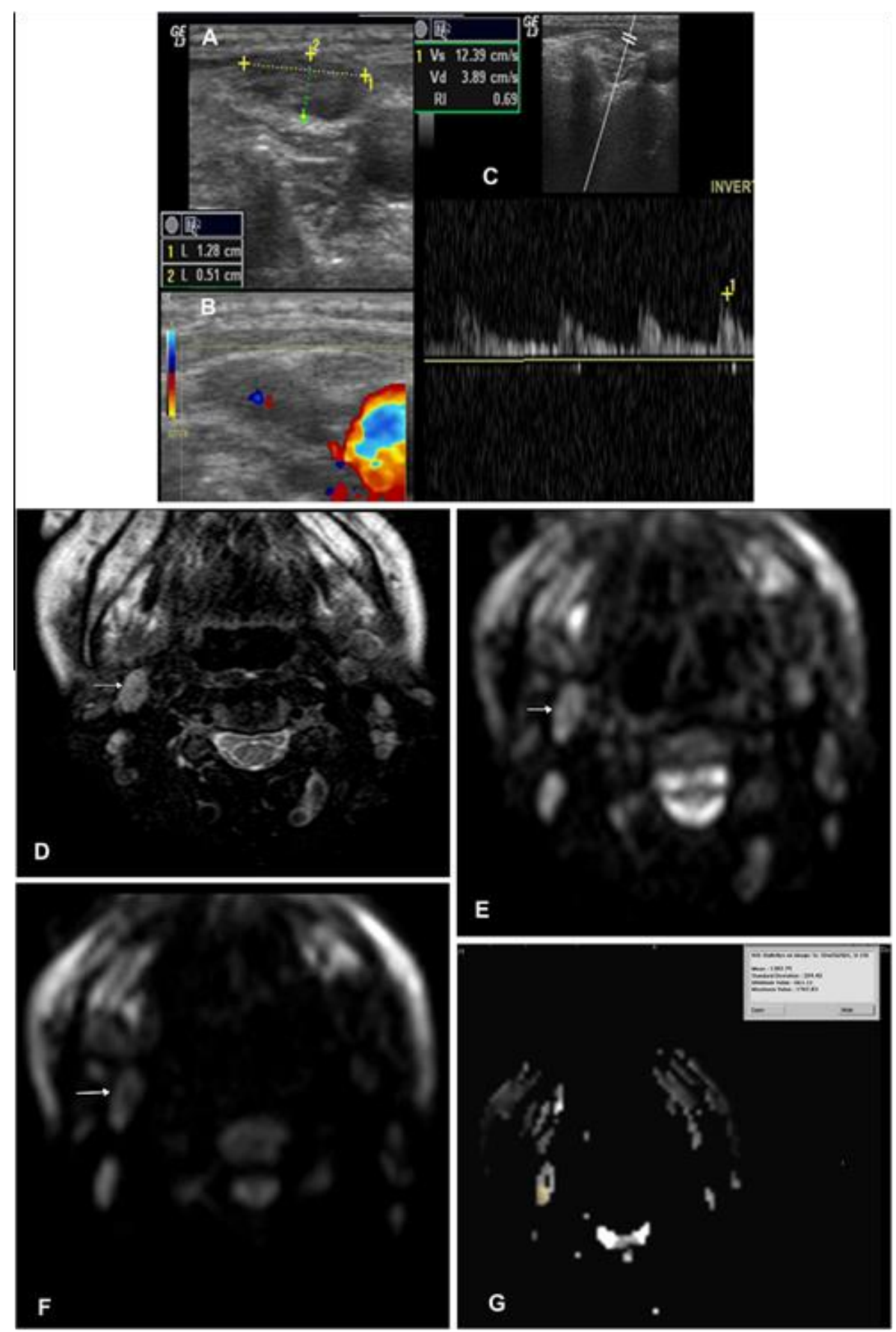

Fig. 4 (A-C) Grey scale, Color and pulsed Doppler US images of the studied L.N; reveal oval shape enlarged LN (S/L ratio $<0.5$ ), and preserved normal central echogenicity with hilar vascularity pattern of perfusion with $\mathrm{RI}=0.69$. (D) Axial STIR-MR image of the studied L.N; reveals enlarged RT upper internal jugular lymph node (arrow) at level IIA that exhibit high signal intensity. (E) DW-MR image at $(\mathrm{b}=0)$ of the studied L.N; shows that the lymph node appear hyperintense (arrow). (F): DW-MR image at $(\mathrm{b}=1000)$ of the studied L.N; shows that the lymph node appears hypointense (arrow). (G) ADC map of the studied L.N; showing high signal intensity of the lymph node. The mean ADC

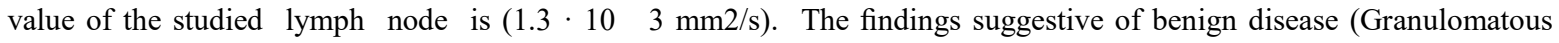
lymphadenopathy due to sarcoidosis). 
Benha medical journal, vol. 39, special issue (radiology), 2022

Table 1 The size and grey scale ultrasound features of the examined lymph nodes

\begin{tabular}{|c|c|c|c|c|}
\hline & Inflammatory $(n=6)$ & Granulomatous ( $n=3$ ) & 1ry Malignant $(n=12)$ & 2ry Malignant $(n=9)$ \\
\hline Short axis length & $\begin{array}{l}5-9.5 \mathrm{~mm} \\
\text { Mean }=7.4 \mathrm{~mm}\end{array}$ & $\begin{array}{l}4-11 \mathrm{~mm} \\
\text { Mean = } 6.4 \mathrm{~mm}\end{array}$ & $\begin{array}{l}8-27 \mathrm{~mm} \\
\text { Mean = } 18 \mathrm{~mm}\end{array}$ & $\begin{array}{l}10-19 \mathrm{~mm} \\
\text { Mean }=15 \mathrm{~mm}\end{array}$ \\
\hline Long axis length & $\begin{array}{l}11-36 \mathrm{~mm} \\
\text { Mean = } 23 \mathrm{~mm}\end{array}$ & $\begin{array}{l}10-29 \mathrm{~mm} \\
\text { Mean = } 19 \mathrm{~mm}\end{array}$ & $\begin{array}{l}18-50 \mathrm{~mm} \\
\text { Mean = 35 mm }\end{array}$ & $\begin{array}{l}14-35 \mathrm{~mm} \\
\text { Mean }=24 \mathrm{~mm}\end{array}$ \\
\hline $\mathrm{S} / \mathrm{L}$ ratio & $\begin{array}{l}0.21-0.66 \\
\text { Mean }=0.42\end{array}$ & $\begin{array}{l}0.30-0.64 \\
\text { Mean }=0.46\end{array}$ & $\begin{array}{l}0.32-0.90 \\
\text { Mean }=0.66\end{array}$ & $\begin{array}{l}0.42-0.82 \\
\text { Mean }=0.63\end{array}$ \\
\hline \multicolumn{5}{|l|}{ Shape } \\
\hline Oval: $\mathrm{S} / \mathrm{L}$ ratio $<0.5$ & $6(100 \%)$ & $2(66.6 \%)$ & $5(41.6 \%)$ & $3(33.3 \%)$ \\
\hline Round: $\mathrm{S} / \mathrm{L}$ ratio $\geqslant 0.5$ & 0 & $1(33.3 \%)$ & $7(58.3 \%)$ & $6(66.6 \%)$ \\
\hline \multicolumn{5}{|l|}{ Hilum } \\
\hline Wide & $5(83.3 \%)$ & $2(66.6 \%)$ & 0 & 0 \\
\hline Narrow & $1(16.6 \%)$ & $1(33.3 \%)$ & $3(25 \%)$ & $2(22.2 \%)$ \\
\hline Absent & 0 & 0 & $9(75 \%)$ & $7(77.7 \%)$ \\
\hline \multicolumn{5}{|l|}{ Echogenicity } \\
\hline Hypoechoic & $6(100 \%)$ & $3(100 \%)$ & $7(58.3 \%)$ & $8(88.8 \%)$ \\
\hline Hyperechoic & 0 & $\mathbf{0}$ & $3(25 \%)$ & $1(11.1 \%)$ \\
\hline Isoechoic & 0 & 0 & $2(6.6 \%)$ & 0 \\
\hline \multicolumn{5}{|l|}{ Homogenicity } \\
\hline Homogenous & $6(100 \%)$ & $3(100 \%)$ & $4(33.3 \%)$ & $2(22.2 \%)$ \\
\hline Heterogeneous & $0(16.6 \%)$ & 0 & $8(66.6 \%)$ & $7(77.7 \%)$ \\
\hline \multicolumn{5}{|l|}{ Borders } \\
\hline Well-defined & $4(66.6 \%)$ & $3(100 \%)$ & $9(75 \%)$ & $7(77.7 \%)$ \\
\hline Ill-defined & $2(33.3 \%)$ & $\mathbf{0}$ & $3(25 \%)$ & $2(22.2 \%)$ \\
\hline \multicolumn{5}{|l|}{ Ancillaryfeafures } \\
\hline Calcification & 0 & $\mathbf{0}$ & $2(16.6 \%)$ & $3(33.3 \%)$ \\
\hline Intranodal Necrosis & 0 & 0 & $8(66.6 \%)$ & $3(33.3 \%)$ \\
\hline Matting & $1(16.6 \%)$ & 0 & $4(33.3 \%)$ & $2(22.2 \%)$ \\
\hline Soft tissue edema & $4(66.6 \%)$ & 0 & 0 & 0 \\
\hline
\end{tabular}

Diffusion weighted MRI (DWMRI) was not better than color Doppler ultrasound (CDUS) in diagnosis of benign lymph nodes because both of them truly diagnosed 8 cases out of the 9 benign cases.

\section{Discussion:}

The evaluation of cervical nodes is important not only for diagnosis and staging of malignant diseases, but also for planning treatment and follow-up (10).

In our study the mean $\mathrm{S} / \mathrm{L}$ ratio for 1ry and 2ry malignant LNs was 0.66 and 0.63 , respectively, which was higher than mean $\mathrm{S} / \mathrm{L}$ ratio of benign $\mathrm{LNs}$ as it was 0.42 for the inflammatory and 0.44 for granulomatous L.Ns. In agreement with other authors (1, 11 and 12), it was stated the $\mathrm{S} / \mathrm{L}$ ratio was a reliable indicator for a metastatic node.

We found preserved wide fatty hilum in $83.3 \%$ of inflammatory LNs and $66.6 \%$ of granulomatous LNs, while absent hilum noticed in most of malignant LNs, which were $75 \%, 77.7 \%$ for 1ry and 2ry malignant LNs, respectively. Similarly in other studies (13 and 14) (13), it was reported that $84 \%$ of benign L.Ns had echogenic hilum. 
In the present study all benign LNs and $71.4 \%(15 / 21)$ of malignant LNs were hypoechoic. Similarly other researches (15 $\& 1$ ), found that echogenicity was unhelpful because the majority of the studied L.Ns involved by disease were hypoechoic.

Calcification and intra-nodal necrosis were found in the malignant L.N either 1ry or 2ry, while none of benign LNs showed these findings (16\&17). But it was not in agreement with those studies ( $1 \& 15)$ which reported that calcifications, necrosis and matting were more frequently seen in benign LNs than in malignant LNs.

Regarding vascular pattern for our studied patients, most of the lymphomatous L.Ns showed mixed flow in 7/12 cases (58.3\%), while metastatic cases showed peripheral flow in 4/9 cases (44.4\%) and mixed in 2/9 cases $(22.2 \%)$. Hilar vascularity was found in all inflammatory lymph nodes and most of granulomatous lymph nodes in $2 / 3$ cases $(66.6 \%)$ Only in one case $(33.3 \%)$ with granulomatous (tuberculous) lymphadenopathy; matted LNs showed mixed vascular pattern of per- fusion. Avascular pattern is found only in one metastatic L.N case (11.1\%). This difference was statistically significant with P-value $=0.004$, and detection of abnormal nodal perfusion patterns (including peripheral, mixed, intranodal dots) showed high sensitivity of $100 \%$ and $76.2 \%$ specificity for malignant L.Ns with PPV $=$ $64.3 \%, \mathrm{NPP}=100 \%$ and $83.3 \%$ accuracy. These results are in agreement with other researcher $(1,12 \& 13)$.

The mean arterial RI for malignant L.Ns in the study was 0.78 , significantly higher than that for benign lymph nodes (0.59) with Pvalue $=0.001$. Mean arterial RI for both inflammatory and granulomatous LNs were as follows $0.55,0.66$, which were significantly lower than that of lymphomatous and metastatic LNs (0.71, 0.86 , respectively) with high statistical significance $(\mathrm{P}-\mathrm{value}=0.000)$. These findings are in agreement with others $(1 \&$ 11), while many authors found that there was no statistically significant difference between RI indices of malignant and benign lymph nodes ( $12 \& 13)$.

We used RI value of $<0.69$ was a cut-off value in differentiation between benign from malignant cervical L.Ns, the resultant accuracy, sensitivity and specificity were as follows $76.7 \%, 88.8 \%$ and $71.4 \%$, in addition cut-off point was found statistically significant $(\mathrm{P}$-value $=$ 0.002). A study done in 1994 (18), reported 
various RI cut-off values among them a RI cut-off value $<0.7$ showed high sensitivity $(81 \%)$ and specificity (75\%) in diagnosing malignant lymph nodes which was in agreement with our study. More specificity of $94 \%$ in distinguishing reactive and metastatic nodes by using an optimal cut-off for $\mathrm{RI}<0.8$ was reported (12). In our study, we used ADC value of $(1.0 \cdot 103$ $\mathrm{mm}^{2} / \mathrm{s}$ ) as a cut-off value in differentiation between benign and malignant cervical lymph nodes, the resultant accuracy, sensitivity and specificity were as follows $96.7 \%$, $100 \%$, and $88.8 \%$, in addition that cut-off point was found to be highly statistically significant cut-off point $(\mathrm{P}$-value $=0.000)$. Similarly a threshold of $1.02 \cdot 103 \mathrm{~mm} 2 / \mathrm{s}$ ADC value was used and 94\% accuracy was recorded in characterizing metastatic lymph nodes with $100 \%$ and $87.0 \%$ for sensitivity and specificity in differentiating between benign and malignant cervical L.Ns (19). On the other hand lower sensitivity and specificity using the same cut off value had reported by other researchers (20), as they reported $92.3 \%$ sensitivity and $83.9 \%$, specificity in their study. Again low ADC threshold for metastatic nodes $(>0.400 \cdot 10 \quad 3$ $\mathrm{mm} 2 / \mathrm{s}$ ) was reported (6) which yielded a moderate negative predictive value 206
$(71 \%)$ and high positive predictive value (93\%), this was not met with our results.

Slightly higher threshold $(\mathrm{ADC}=1.38 \cdot 10$ $3 \mathrm{~mm} 2 / \mathrm{s}$ ) for reliably characterizing suspected malignant lymph nodes with an accuracy of $96 \%$, sensitivity of $98 \%$ and specificity of $88 \%$ (8). Moreover, the mean ADC value for benign nodes either inflammatory or granulomatous nodes (1.56 - $103-1.448 \cdot 103 \mathrm{~mm} 2 / \mathrm{s})$, was higher compared to the mean ADC value of metastatic and lymphomatous nodes.

We found also that the Non Hodgkin lymphomatous nodes had lower ADC values (mean $0.69 \cdot 10 \quad 3 \mathrm{~mm}^{2} / \mathrm{s}$ ) than Hodgkin lymphomatous nodes (mean $0.81 \cdot 103 \mathrm{~mm}^{2} / \mathrm{s}$ ), however this difference was not statistically significant because Pvalue $=0.12$. Similarly Steinkamp et al. (12) and Ali (21) found that the mean ADC of non-Hodgkin lymphoma is 0.93 - $103 \mathrm{~mm}^{2} / \mathrm{s}$ usually lower than the mean ADC of Hodgkin lymphoma (1.03 - $\left.103 \mathrm{~mm}^{2} / \mathrm{s}\right)$.

Using histopathological examination as the standard reference; we found that diffusion weighted MRI and ADC was capable of diagnosing all histologically proven malignant nodes, while it diagnosed 8 out of the 9 histologically proven benign 
nodes. In one case of sarcoidosis the studied lymph node showed relatively low ADC value $\left(0.9 \cdot 103 \mathrm{~mm}^{2} / \mathrm{s}\right)$ this was in agreement with other studies (8 and 22) which reported that granulomatous lesions, such as sarcoid and tuberculous nodes, may exhibit low ADC values.

Table 2 Vascular Pattern of the Lymph Nodes in the Studied Cases.

\begin{tabular}{lllll}
\hline Vascular pattern & $\begin{array}{l}\text { Inflammatory } \\
(n=6)\end{array}$ & $\begin{array}{l}\text { Granulomatous } \\
(n=3)\end{array}$ & $\begin{array}{l}\text { 1ry Malignant } \\
(\text { lymphoma })(n=12)\end{array}$ & $\begin{array}{l}\text { 2ry Malignant } \\
(\text { metastasis })(n=9)\end{array}$ \\
\hline Hilar & $6(100 \%)$ & $2(66.6 \%)$ & $1(8.3 \%)$ & 0 \\
Peripheral & 0 & 0 & $3(25 \%)$ & $4(44.4)$ \\
Mixed & 0 & $1(33.3 \%)$ & $7(58.3 \%)$ & $2(22.2 \%)$ \\
Intranodal dots & 0 & 0 & $1(8.3 \%)$ & $2(22.2 \%)$ \\
Avascular & 0 & 0 & 0 & $1(11.1 \%)$ \\
\hline
\end{tabular}

Table 3 The distribution of apparent diffusion coefficient (ADC) values among the benign and malignant lymph nodes.

\begin{tabular}{llll}
\hline Apparent diffusion coefficient (ADC) values & Benign & Malignant & $P$-value \\
\hline Range & $\left(0.93 \times 10-2.13 \times 10^{-3} \mathrm{~mm}^{2} / \mathrm{s}\right)$ & $\left(0.59 \times 10-0.98 \times 10^{-3} \mathrm{~mm}^{2} / \mathrm{s}\right)$ & $0.000^{* * *}$ \\
Mean & $1.42 \times 10^{-3} \mathrm{~mm}^{2} / \mathrm{s}$ & $0.78 \times 10^{-3} \mathrm{~mm}^{2} / \mathrm{s}$ & .
\end{tabular}

ADC values are measured by ROI placed on solid nodal regions; necrotic regions (which were found in some of lymphomatous and metastatic $\mathrm{LNs}$ ) are excluded from these calculations and analyzed later.

${ }_{* * *}^{*}=$ Statistically significant, as $P$-value less than 0.05 .

\section{Conclusion:}

The use of DWI and ADC was useful for differentiation between benign and malignant cervical lymphadenopathy and recommended to decrease the need of invasive biopsies.

\section{References}

(1) Gupta A, Rahman K, Shahid M, Kumar A, Qaseem SMD, Hassan S, et al. Sonographic assessment of cervical lymphade- nopathy: role of high-resolution and color Doppler imaging. Head Neck 2011;33:297-302.

(2) Adibelli ZH, Unal G, Gul E, Uslu F, Kocak U, Abali Y. Differentiation of benign and malignant cervical lymph nodes: value of B-mode and color Doppler sonography. Eur J Radiol 1998;28:230-4.

(3) Castelijns JA, Van den Brekel MW. Imaging of lymphadenopathy in the neck. Eur Radiol 2002;12:727-38.

(4) Steinkamp HJ, Mueffelmann M, Bock JC, Thiel T, Kenzel P, Felix R. Differential diagnosis of lymph node lesions: a semi- quantitative approach with color Doppler ultrasound. Br J Radiol 1998;71:828-33.

(5) Kwee TC, Takahara T, Luijten PR, Nievelstein RA. ADC measurements of lymph nodes: inter- and intra-observer repro- ducibility study and an overview of the literature. Eur $\mathbf{J}$ Radiol 2010;75:215-20.

(6) Sumi M, Sakihama N, Sumi T, Morikawa M, Uetani M, Kabasawa $\mathrm{H}$, et al. Discrimination 
of metastatic cervical lymph nodes with diffusionweighted MR imaging in patients with head and neck cancer. Am J Neuroradiol 2003;24:1627-34.

(7) Sumi M, Van Ceuteren M, Nakamura T. MR microimaging of benign and malignant nodes in the neck. AJR Am J Roentgenol 2006;186:749-57.

(8) Razek AA, Soliman NY, Elkhamary S, Alsharaway MK, Tawfik A. Role of diffusionweighted MR imaging in cervical lymphadenopathy. Eur Radiol 2006;16:1468-77.

(9) Kwee TC, Takahara T, Ochiai R, Nievelstein RAJ, Luijten P. Diffusion weighted whole-body imaging with background body signal suppression (DWIBS): features and potential applications in oncology. Eur J Radiol 2008;18:1952-73.

(10) Perrone A, Guerrisi P, Izzo L, D’Angelic I, Sassi S, Mele LL, et al. Diffusion-weighted MRI in cervical lymph nodes: differen- tiation between benign and malignant lesions. Eur J Radiol 2011; 77:281-6.

(11) Na DG, Lim HK, Byun HS, Kim HD, Ko YH, Baek JH. Differential diagnosis of cervical lymphadenopathy: usefulness of color Doppler sonography. AJR Am Roentgen 1997;168:1311-6.

(12) Steinkamp HJ, Maurer J, Cornehl M, Knobber D, Hettwer H, Felix R. Recurrent cervical lymphadenopathy: differential diagnosis with color-duplex sonography. Eur Arch Otorhinolar- yngol 1994;251:404-9.

(13) Rubaltelli L, Proto E, Salmaso R, Bortolett P, Candiani F, Cagol P. Sonography of abnormal lymph nodes in vitro: correlation of sonographic and histologic findings. AJR Am J Roentgenol 1990; 155:1241-4.

(14) Ahuja A, Ying M. Sonographic evaluation of cervical lymph nodes. AJR 2005;184:1691-9.

(15) Ying M, Ahuja AT, Evans R, King W, Metreweli C. Cervical lymphadenopathy: sonographic differentiation between tubercu- lous nodes and nodal metastases from non-head and neck carcinomas. J Clin Ultrasound 1998;26:383-9.

(16) Ahuja A, Ying M. Grey-scale sonography in assessment of cervical lymphadenopathy: review of sonographic appearances and features that may help a beginner. Br J Oral Maxillofac Surg 2000;38:451-9.

(17) Ying M, Ahuja A, Brook F. Sonographic appearances of cervical lymph nodes: variations by age and sex. J Clin Ultrasound 2000; 30:1-11.

(18) Chang DB, Yuan A, Yu CJ, Luh KT, Kuo $\mathrm{SH}$, Yang PC. Differentiation of benign and malignant cervical lymph nodes with color Doppler sonography. A J Roentgen 1994;162:9658.

(19) Holzapfel K, Duetsch S, Fauser C, Eiber M, Rummeny EJ, Gaa J. Value of diffusion-weighted MR imaging in the differentiation between benign and malignant cervical lymph nodes. Eur J Radiol 2009;72:381-7.

(20) De Bondt RB, Nelemans PJ, Bakers F, Casselman JW, Peutz- Kootstra C, Kremer B, et al. Morphological MRI criteria improve the detection of lymph node metastases in head and neck squamous cell carcinoma: multivariate 
logistic regression analysis of MRI features of cervical lymph nodes. Eur Radiol

2009;19:626-33.

(21) Ali T. Neck lymph nodes: characterization with diffusion- weighted MRI. Egypt Soc Radiol Nucl Med 2012;43(2):17381.

To cite this article: Mohamed A. Mohammad, Tamer A. Kamal, Haney H. Lotfy Benign Versus Malignant Cervical Lymph Nodes ; Differentiation by Diffusion Weighted MRI. BMFJ 2022;39 (Radiology):194-209. DOI: 10.21608/bmfj.2021.21437.1196 